\title{
Re-Entry of Space Objects from Low Eccentricity Orbits
}

\author{
Cynthia Sharon Lawrence, Ram Krishan Sharma \\ Department of Aerospace Engineering, Karunya Institute of Technology and Sciences, Coimbatore, India \\ Email: cynthiasatlaw@gmail.com,ramkrishansharma@gmail.com
}

How to cite this paper: Lawrence, C.S. and Sharma, R.K. (2019) Re-Entry of Space Objects from Low Eccentricity Orbits. International Journal of Astronomy and Astrophysics, 9, 200-216. https://doi.org/10.4236/ijaa.2019.93015

Received: April 29, 2019

Accepted: August 27, 2019

Published: August 30, 2019

Copyright ( 2019 by author(s) and Scientific Research Publishing Inc. This work is licensed under the Creative Commons Attribution International License (CC BY 4.0).

http://creativecommons.org/licenses/by/4.0/

(c) (i) Open Access

\begin{abstract}
This paper deals with the re-entry predictions of the space objects from the low eccentric orbit. Any re-entering object re-enters the Earth's atmosphere with a high orbital velocity. Due to the aerodynamic heating the object tends to break into multiple fragments which later pose a great risk hazard to the population. Here a satellite is considered as the space object for which the re-entry prediction is made. This prediction is made with a package where the trajectory path, the time of re-entry and the survival rate of the fragments is done. The prediction is done using DRAMA 2.0-ESA's Debris Risk Assessment and Mitigation Analysis Tool suite, MATLAB and Numerical Prediction of Orbital Events software. The predicted re-entry time of OSIRIS $3 \mathrm{U}$ was found to be on 7th March 2019, 7:25 (UTC), whereas the actual re-entry time was on 7th March 2019, 7:03 (UTC). The trajectory path found was 51.5699 deg. (Lat), -86.5738 deg. (Long.) with an altitude of $168.643 \mathrm{~km}$. But the actual trajectory was $51.76 \mathrm{deg}$. (Lat), $-89.01 \mathrm{deg}$. (Long.) with an altitude of $143.5 \mathrm{~km}$.
\end{abstract}

\section{Keywords}

Re-Entry, Space Objects, Low Eccentricity Orbits, DRAMA 2.0, Risk Event Statistics

\section{Introduction}

Space objects refer to astronomical objects as well as the artificial space objects, i.e. naturally occurring or man-made objects in space. Both the astronomical and artificial objects tend to enter the Earth's atmosphere, especially the man-made objects. Man-made objects like satellites and rockets are being sent to space for communication, navigation and other space missions. They tend to decay after a certain orbital lifetime. Spent rocket stages, old satellites and fragments from 
disintegration, erosion and collisions are considered as debris. In particular, an accurate estimation of orbital decay of objects during the final stages of re-entry is of considerable importance. This helps to predict the re-entry time and location and thus plan proper hazard assessment and mitigation strategies. The database available for the prediction of orbital lifetime and re-entry of debris objects is the set of two line elements (TLEs). In general, the physical parameters of the objects like mass, area of cross section, shape and dimensions are not available accurately. Further, the atmosphere in which the objects decay varies significantly. A low Earth orbit lies between the altitudes 150 and 2000 kilometer, with a period of about 88 minutes to 127 minutes. Some of the important studies carried out in the area reported in this study are in References [1]-[11].

In this paper, a method to carry out the re-entry predictions of space debris entering from low eccentricity Earth orbit, employing the orbital data in the form of TLEs, is presented. The ballistic coefficient and eccentricity of the re-entering objects are considered as uncertain parameters. The Earth's zonal harmonic terms $J_{2}$ to $J_{6}$ are included along with the drag perturbation [1] [2].

The re-entry time estimation in each case is computed using NPOE software and MATLAB. The influence of luni-solar perturbations, Earth's oblateness and atmospheric drag are considered to predict the re-entry time. The re-entry trajectory path along with the debris risk analysis is determined using Debris Risk Assessment and Mitigation Analysis (DRAMA) software. The orbital data and other details of the space objects which re-enter are taken from space-track.org maintained by US Air Force.

NPOE is an interactive computer program for computers which can model important orbital events and predict the long-term evolution of satellites in Earth orbits. Program NPOE implements a special perturbation solution of orbital motion using a variable step size Runge-Kutta-Fehlberg (RKF78) integration method to numerically integrate Cowell's form of the system of differential equations. Orbital events are predicted using Brent's method for finding the root of a nonlinear equation.

MATLAB has also been used to predict the long-term behaviour of the Earth's satellites subjected to various perturbations. The program actualizes a special perturbation solution of orbital motion as same as NPOE using the variable step size Runge-Kutta-Fehlberg (RKF78) integration method to numerically solve Cowell's form of the system of differential equation subjected to the central body gravity and other external forces which is otherwise called as orbital initial value problem (IVP).MATLAB is used to plot the graphs of the orbital events.

DRAMA is a comprehensive tool [3] for the compliance analysis of space mission with space debris mitigation standards provides with distinct tools to enable the assessment of debris mitigation strategies for the operational and disposal phases of a mission as well as the estimation of the risk caused by objects surviving a re-entry of the spacecraft.

The following tools that are available within DRAMA are: 
1) ARES: Assessment of Risk Event Statistics

The ARES provides an assessment of collision-related events between an operational spacecraft and trackable objects orbiting the Earth, the statistical collision probability, the mean number of conjunction avoidance manoeuvres and the fuel consumption associated to those manoeuvres.

2) MIDAS: MASTER (Based) Impact Flux and Damage Assessment

In MIDAS user-defined BLEs can be provided and flux computations are now performed using the MASTER-2009 model. Debris and meteoroid collision flux and damage analysis are done using MIDAS.

3) OSCAR: Orbital Spacecraft Active Removal

OSCAR offers the possibility to select between different standardized methods (ISO, ECSS) to generate forecasts of future solar and geomagnetic activity. OSCAR allows for the simulation of drag augmentation devices as a new disposal system.

4) CROC: Cross Section of Complex Bodies

Computes the cross-section of complex bodies for different aspect angle conditions.

5) SARA: (Re-Entry) Survival and Risk Analysis

It is used for the simulation of the re-entry into the Earth's atmosphere.

This prediction package is used to study the re-entry of OSIRIS $3 \mathrm{U}$ satellite. OSIRIS-3U is a CubeSat mission launched on 14 August 2017. Orbital Satellite for Investigating the Response of the Ionosphere to Stimulation and Space Weather is the acronym of OSIRIS which is a three-unit CubeSat developed by the students of the Penn State University. The mission of OSIRIS-3U is to investigate the radio wave interaction in the ionosphere, particularly the interaction of high-power radio waves.

\section{Working with NPOE and MATLAB}

Newton's law [4] describes the force between two bodies of masses acting on each other that are at a particular distance. The same theory is used on the two-body problem, where the earth has a bigger body mass than the satellite. The law of gravitation is used here is as,

$$
F=\frac{-G M m}{r^{2}}
$$

where,

$G=6.673 \times 10^{-11} \mathrm{~m}^{3} \cdot \mathrm{kg}^{-1} \cdot \mathrm{s}^{-2}$, the gravitational constant;

$M=$ the mass of centre object;

$m=$ the mass of orbiting object;

$r=$ the distance between the two masses.

Transforming into the vector form,

$$
\ddot{r}=\frac{-G M}{r^{3}} r
$$

The mass of the satellite $(m)$ is neglected due to its small mass. 
In this study eccentricity and ballistic coefficient are considered as uncertain parameters [5]. The ballistic coefficient depends on the mass of the object, drag coefficient and the effective area. Of these, drag coefficient and effective area have more significant uncertainties. For the ballistic coefficient $\left(M=m / C_{d} A\right)$, the drag coefficient $\left(C_{d}\right)$ and the drag area are $2.20 .03 \mathrm{~m}^{2}[6]$.

As mentioned in the introduction, NPOE and MATLAB script was used to implement a special perturbation solution of orbital motion using a variable step size Runge-Kutta-Fehlberg (RKF78) integration method to numerically solve Cowell's form of the system of differential equation subject to the central body gravity and other external forces.

$$
\boldsymbol{a}(\boldsymbol{r}, \boldsymbol{v}, t)=\ddot{\boldsymbol{r}}(\boldsymbol{r}, \dot{\boldsymbol{r}}, t)=\boldsymbol{a}_{g}(\boldsymbol{r})+\boldsymbol{a}_{d}(\boldsymbol{r}, \boldsymbol{v}, t)+\boldsymbol{a}_{s m}(\boldsymbol{r}, t)+(\boldsymbol{r}, t)
$$

The satellite's acceleration due to Earth's gravity field is calculated with a vector equation derived from the gradient of the potential function expressed as

$$
\boldsymbol{a}_{g}(\boldsymbol{r}, t)=\nabla \phi(\boldsymbol{r}, t)
$$

This acceleration vector is a combination of pure two-body or point mass gravity acceleration and the gravitational acceleration due to higher-order non-spherical terms in the Earths geo-potential.

The acceleration experienced by the satellite due to atmospheric drag [7] is computed in NPOE using the following vector expression:

$$
\boldsymbol{a}_{d}(\boldsymbol{r}, \boldsymbol{v}, t)=-\frac{1}{2} \rho(\boldsymbol{r}, t)\left|\boldsymbol{v}_{r}\right| \boldsymbol{v}_{r} \frac{C_{d} A}{m}
$$

The acceleration contribution of the Sun and Moon represented by point masses is given by

$$
\boldsymbol{a}_{s m}(\boldsymbol{r}, t)=-\mu_{m}\left(\frac{\boldsymbol{r}_{m-b}}{\left|\boldsymbol{r}_{m-b}\right|^{3}}+\frac{\boldsymbol{r}_{e-m}}{\left|\boldsymbol{r}_{e-m}\right|^{3}}\right)-\mu_{s}\left(\frac{\boldsymbol{r}_{s-b}}{\left|\boldsymbol{r}_{s-b}\right|^{3}}+\frac{\boldsymbol{r}_{e-s}}{\left|\boldsymbol{r}_{e-s}\right|^{3}}\right)
$$

Generally, in the low earth orbit, the space objects are more prone to the gravity and atmospheric drag. But when the altitude exceeds approximately $1000 \mathrm{~km}$, the solar effects from the Sun increase. Here, OSIRIS $3 \mathrm{U}$ is at an altitude between $180 \mathrm{~km}-210 \mathrm{~km}$ and so the solar radiation pressure along with the luni-solar perturbation is being neglected.

In this case, the variation of re-entry time is determined based on varying the ballistic coefficient (BC) [8] and the following graphs Figures 1-4 have been plotted, respectively.

It is found based on the data plotted (Figures 1-4) that the ballistic coefficient does not show much variation in the re-entry time as expected. The graphs show that the orbital lifetime with $\mathrm{BC}=60 \mathrm{~kg} / \mathrm{m}^{2}$ and $\mathrm{BC}=80 \mathrm{~kg} / \mathrm{m}^{2}$ show very less variation.

Hence, it was neglected as being an uncertain parameter and $\mathrm{BC}=70 \mathrm{~kg} / \mathrm{m}^{2}$ was taken for the prediction of re-entry time of OSIRIS 3U. The observed semi-major axis and the osculating eccentricity from the TLEs are given in Table 1. 


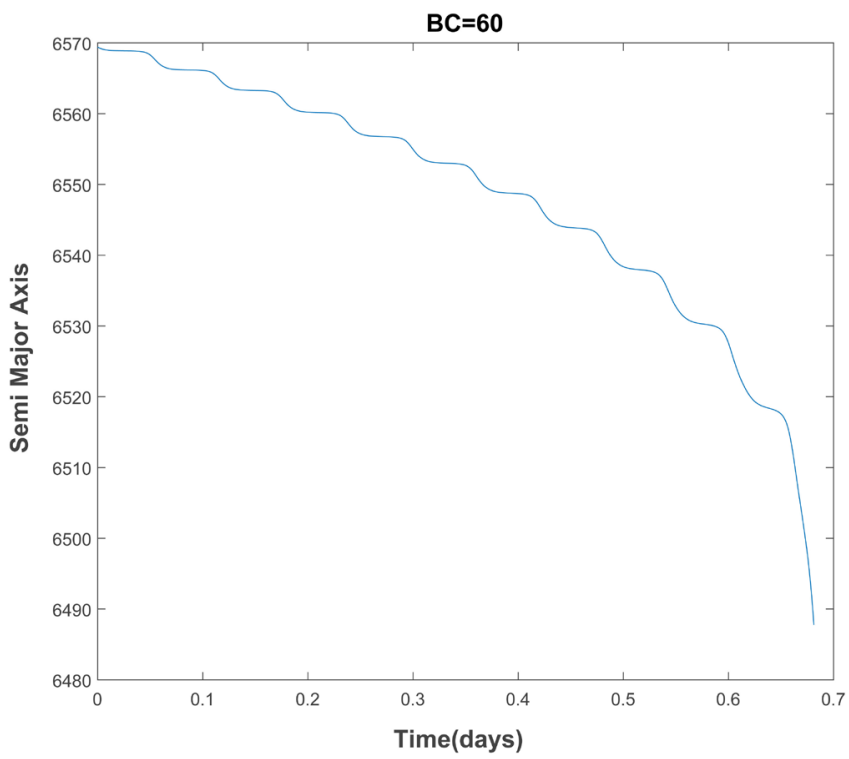

Figure 1. Change in semi major axis for $\mathrm{BC}=60 \mathrm{~kg} / \mathrm{m}^{2}$.

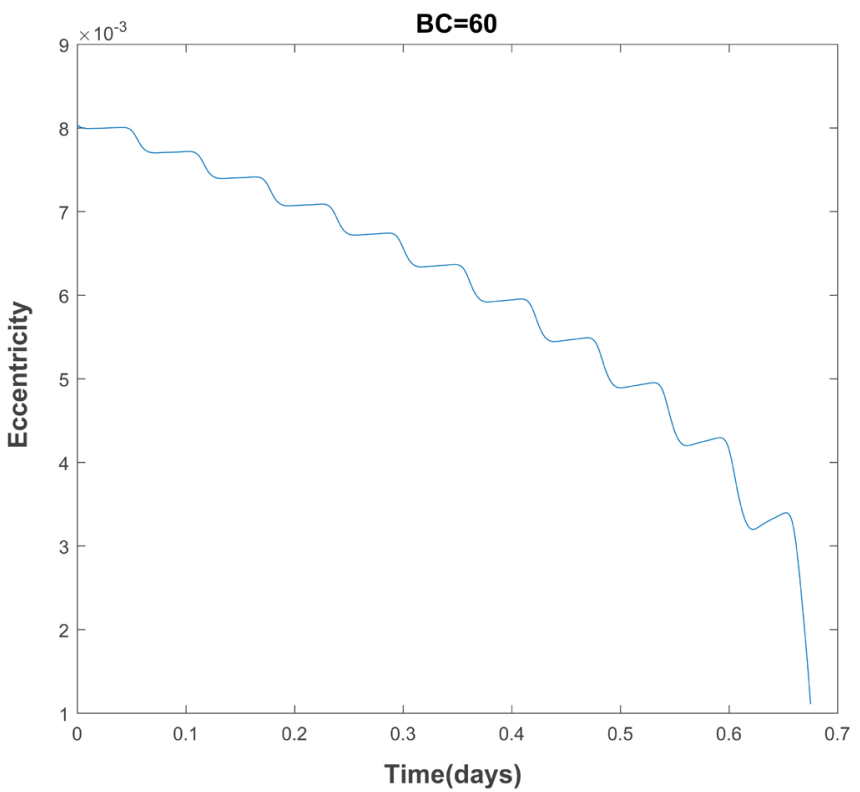

Figure 2. Change in eccentricity for $\mathrm{BC}=60 \mathrm{~kg} / \mathrm{m}^{2}$.

Table 1. Osculating orbital elements.

\begin{tabular}{ccc}
\hline TLE Epoch (UTC) & Semi Major Axis (km) & Eccentricity \\
\hline $04.02 .1920: 31$ & 6669.964476 & 0.0005899031 \\
$09.02 .1913: 25$ & 6662.310370 & 0.0002481707 \\
$10.02 .1921: 01$ & 6664.590117 & 0.0004735118 \\
$11.02 .1922: 34$ & 6661.128219 & 0.0007581420 \\
$13.02 .1921: 10$ & 6656.298443 & 0.0011430093 \\
$15.02 .1919: 43$ & 6653.712256, & 0.0011598911 \\
$18.02 .1919: 44$ & 6641.503586 & 0.0012821558 \\
$22.02 .1912: 08$ & 6649.046280 & 0.0011109638
\end{tabular}




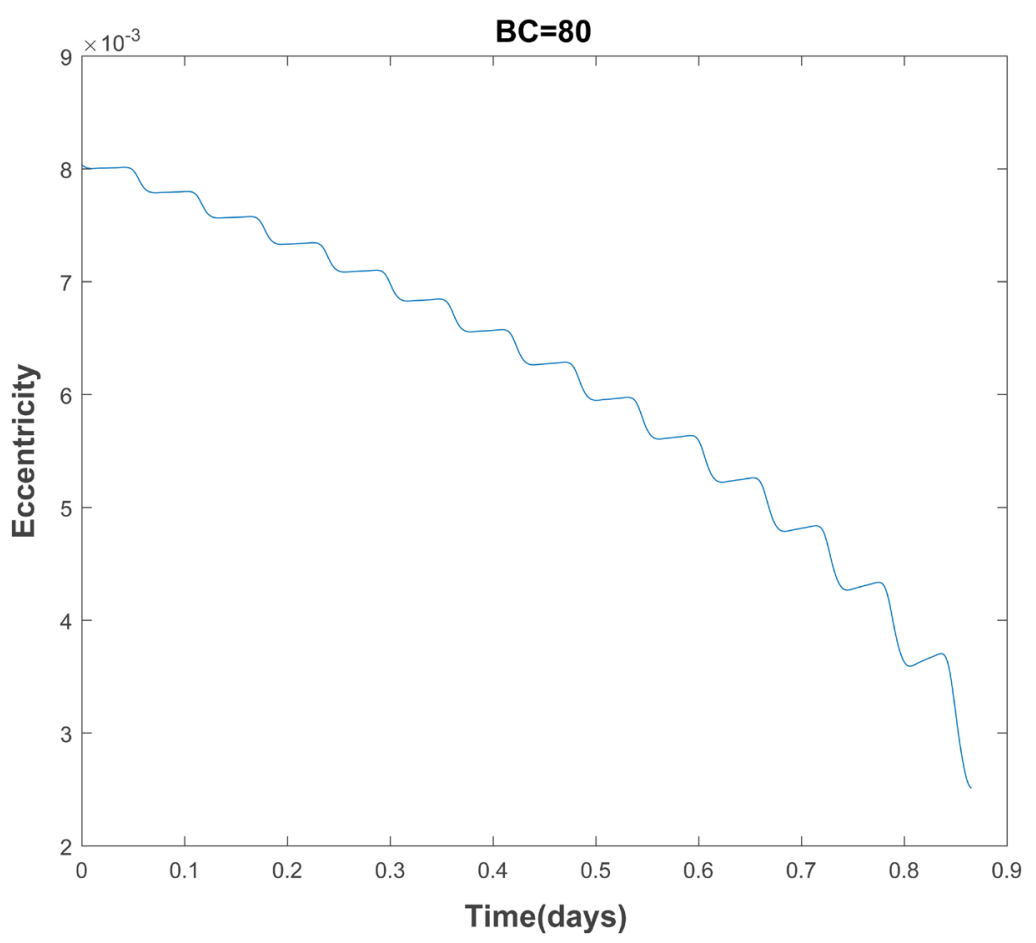

Figure 3. Change in eccentricity for $\mathrm{BC}=80 \mathrm{~kg} / \mathrm{m}^{2}$.

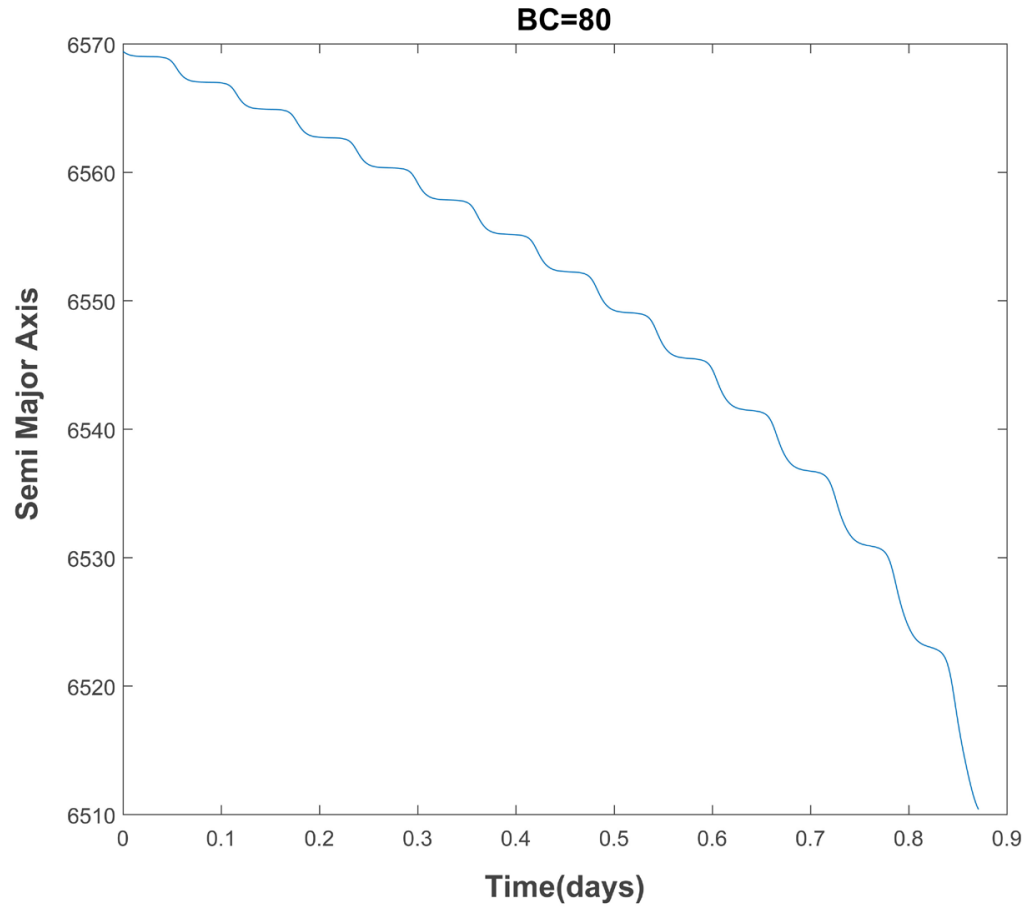

Figure 4. Change in semi major axis for $\mathrm{BC}=80 \mathrm{~kg} / \mathrm{m}^{2}$.

The osculating eccentricity and ballistic coefficient taken as uncertain parameters were found to be 0.0080354104 and $70 \mathrm{~kg} / \mathrm{m}^{2}$, respectively. Using the estimated values, the re-entry time was predicted to be on 7th March 7:25 (UTC) as shown in the graphs (Figure 5 and Figure 6). 


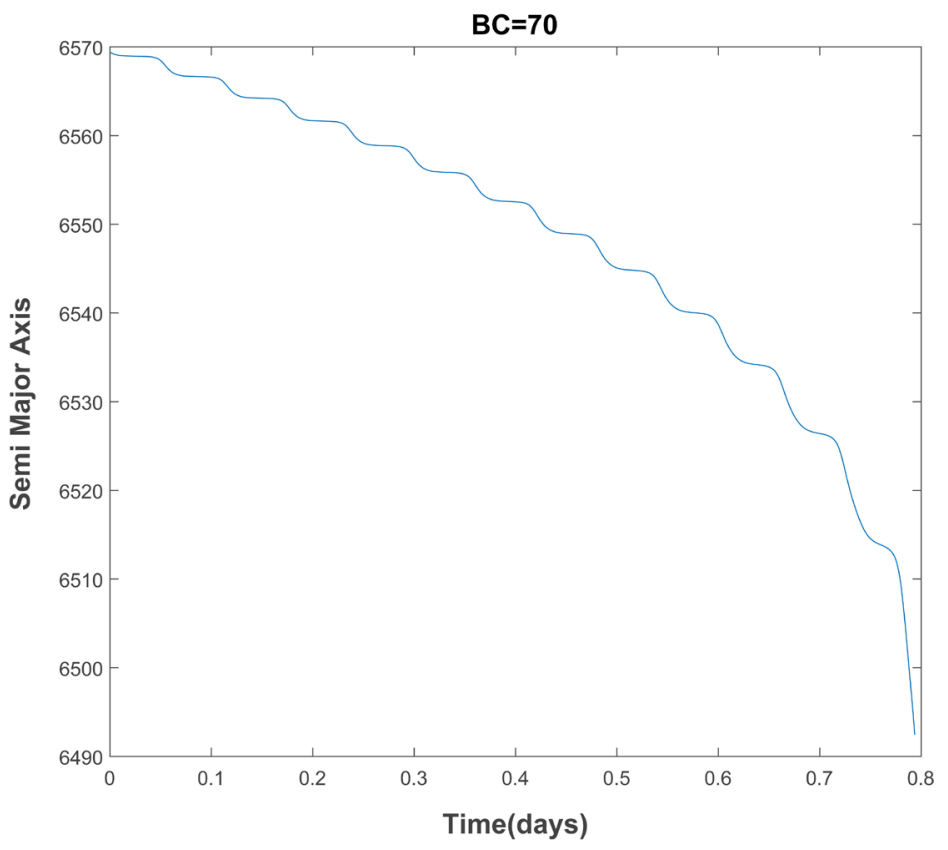

Figure 5. Change in semi major axis for $\mathrm{BC}=70 \mathrm{~kg} / \mathrm{m}^{2}$.

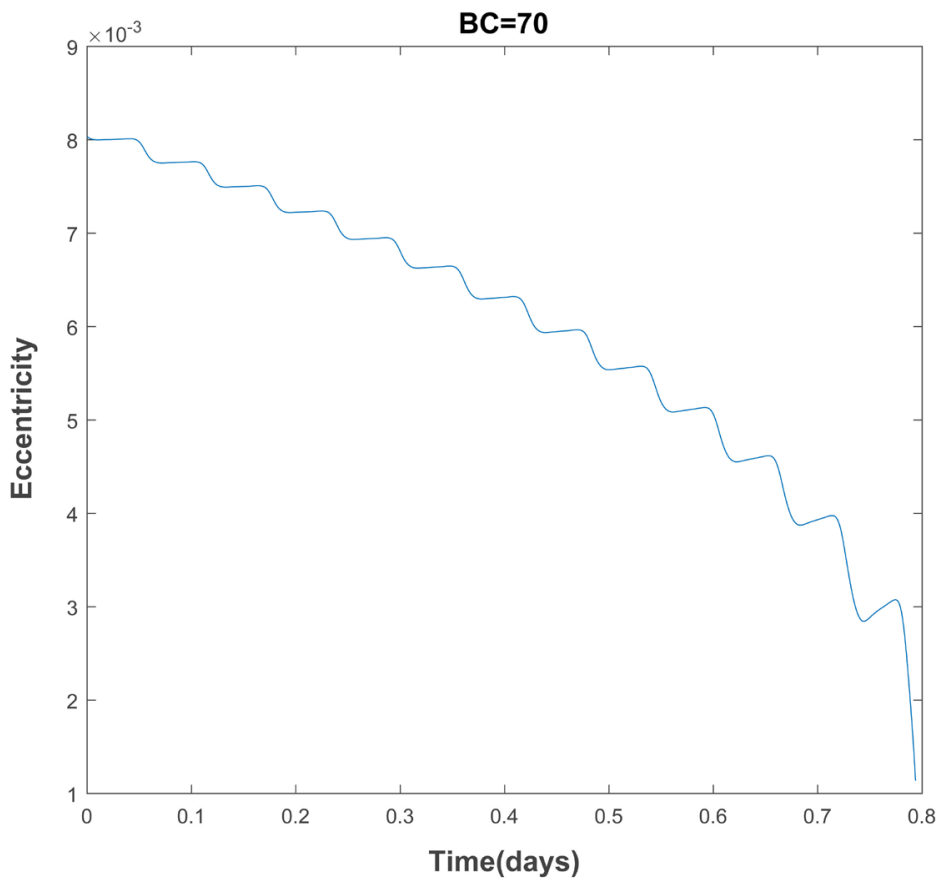

Figure 6. Change in eccentricity for $\mathrm{BC}=70 \mathrm{~kg} / \mathrm{m}^{2}$.

\section{Working with Drama}

\subsection{ARES}

\subsubsection{Annual Collision Probability (ACP)}

The Annual Collision Probability (ACP) [3] is modelled by means of an analogy with the laws of kinetic gas theory. The mean number of collisions encountered by an object with a collision section $A_{c}$ which moves through a stationary me- 
dium of uniform particle density $D$, at a constant velocity $v$ during a given time $\Delta t$ is expressed as:

$$
c=v D A_{c} \Delta t
$$

When simulation was performed on OSIRIS $3 \mathrm{U}$ with the ARES tool for the ACP functionality, the functionality returned with the value as in Table 2.

Table 2. ACP.

\begin{tabular}{cc}
\hline Functionalities & Values \\
\hline ACP_d & $0.7141 \mathrm{E}-05$ \\
ACP_w & $0.6424 \mathrm{E}-03$ \\
Flux_d & $0.6910 \mathrm{E}-01$ \\
Flux_w & $0.1011 \mathrm{E}+02$ \\
\hline
\end{tabular}

where, ACP_w - probability of collision with any object of the whole population; $\mathbf{A C P}$ _d - probability of collision with detectable objects; Flux_d_-flux due to the detected population $\left[1 / \mathrm{km}^{2} /\right.$ year]; Flux_w - flux due to the whole population $\left[1 / \mathrm{km}^{2} /\right.$ year $]$.

\subsubsection{Avoidance Schemes Assessment}

\section{Mean number of Manoeuvres per Year}

The probability of collision for an object-to object encounter is computed by

$$
P=\frac{1}{2 \pi \sqrt{\operatorname{det}(C)}} \int_{-R}^{R} \int_{-\sqrt{R^{2}-x^{2}}}^{\sqrt{R^{2}-x^{2}}} \mathrm{e}^{-\frac{1}{2} \delta r^{\mathrm{T}} C^{-1} \delta r} \mathrm{~d} y \mathrm{~d} x
$$

where,

$R$-sum of the two object radii;

$\delta \boldsymbol{r}$-vector between a point in the integration area and the point where the near-miss is predicted.

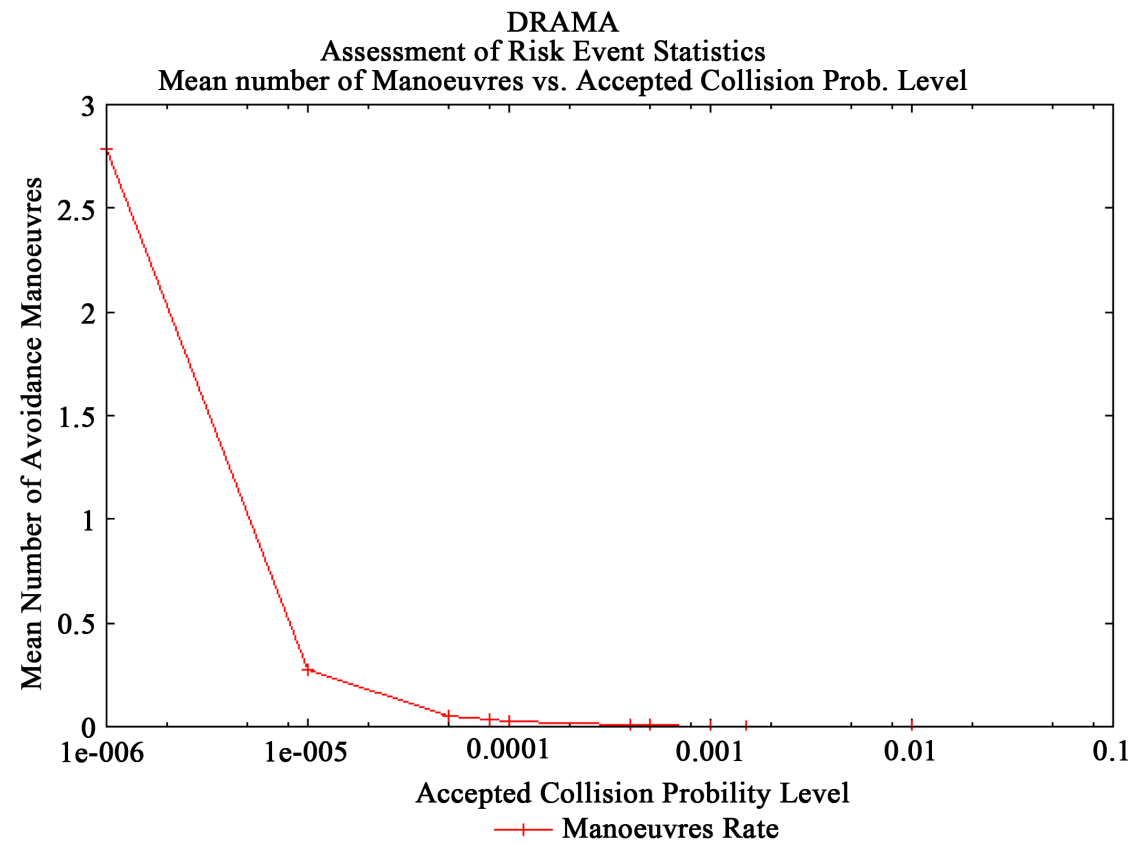

Figure 7. Predicted manoeuvres per year. 
Figure 7 shows the yearly mean number of avoidance manoeuvres predicted for OSIRIS 3U. The number of manoeuvres is a function of accepted collision probability level. According to the same figure, if OSIRIS 3U operators wanted to avoid all collisions with a probability greater than 1e-006, they would need to perform approximately 2.8 manoeuvres.

\section{Risk Reduction and False Alarm Rate}

Figure 8 and Figure 9, shows how much the number of manoeuvres contributes to mitigating the collision risk. If OSIRIS $3 \mathrm{U}$ performs the 2.8 manoeuvres that are required to prevent all close encounters with a probability of collision greater than 1e-006, the collision risk would approximately be ACP_w - ACP_d (Table 2).

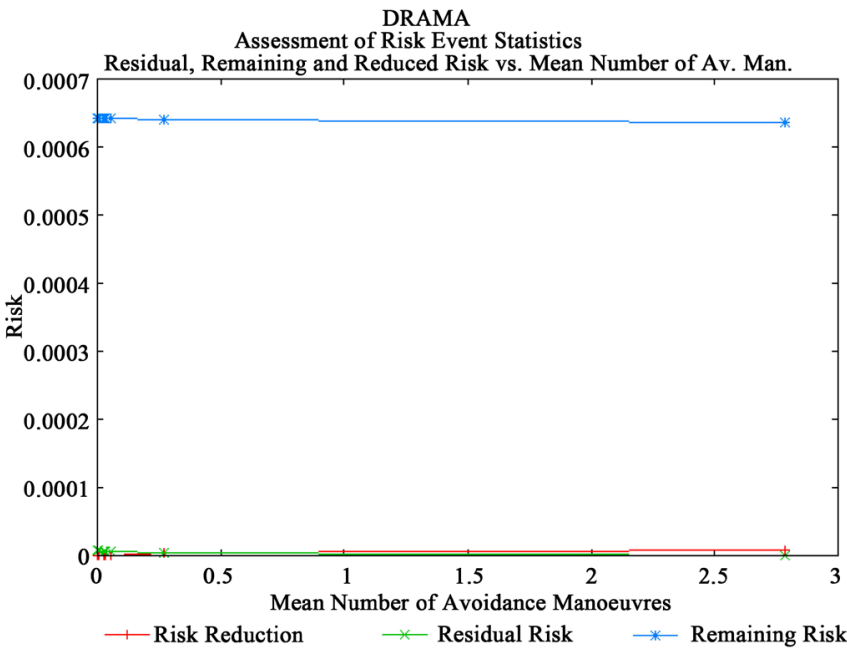

Figure 8. Risk for OSIRIS 3U.

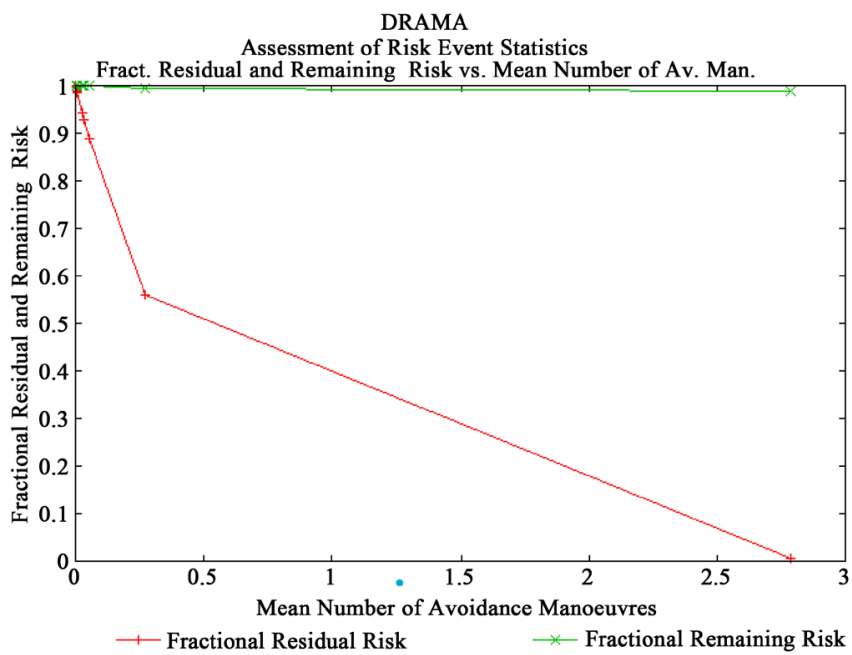

Figure 9. Remaining risk for OSIRIS 3 U.

Finally, Figure 10 shows the accepted collision probability level along with the false alarm rate. False Alarm rates are high, due to the uncertain position of the objects that are involved in the collisions. 


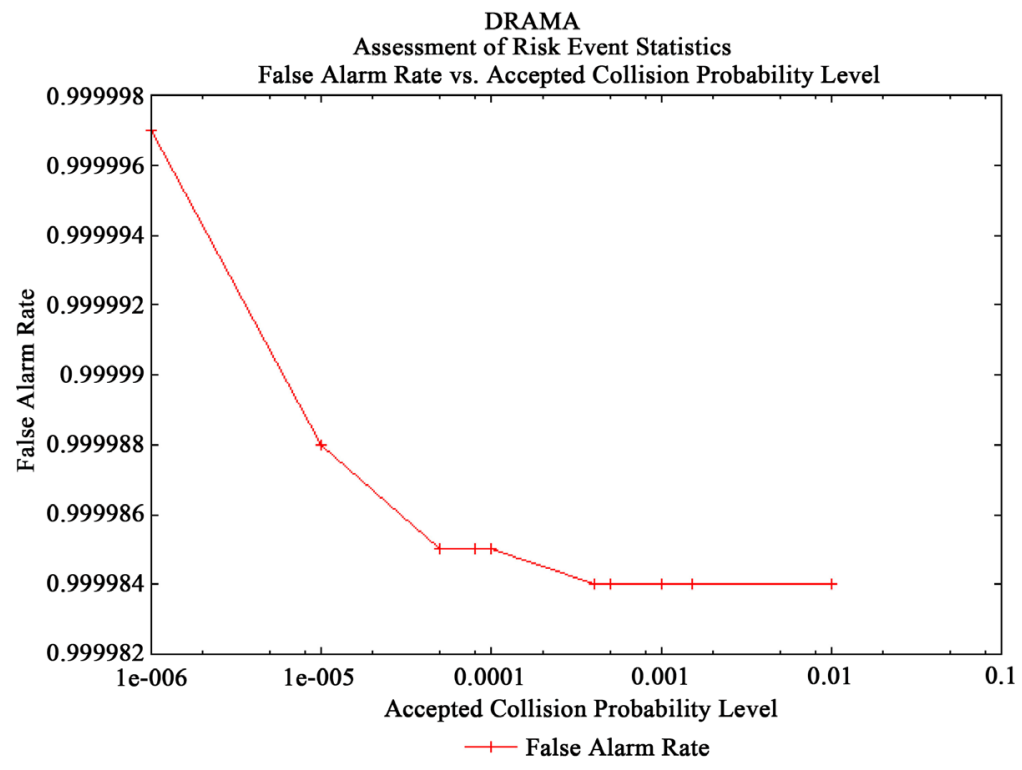

Figure 10. False alarm rate for OSIRIS $3 \mathrm{U}$.

\subsubsection{Required $\Delta V$}

The required $\Delta V_{j}$ (for each population group) is obtained by integration of $\Delta V$ $F_{j}$ over the manoeuvring area. The total $\Delta V\left(\Delta V_{T}\right)$ is obtained by adding the contribution of each population group:

$$
\Delta V_{T}=\sum_{j} \Delta V_{j}
$$

Figure 11 shows how much of $\Delta V$ is required to reach the ACPL values. The $\mathrm{x}$-axis represents the collision avoidance strategy. A value of 0 means a cross-track manoeuvre, while values greater than 0 represents along-track manoeuvres.

DRAMA

Assessment of Risk Event Statistics Delta-Velocity

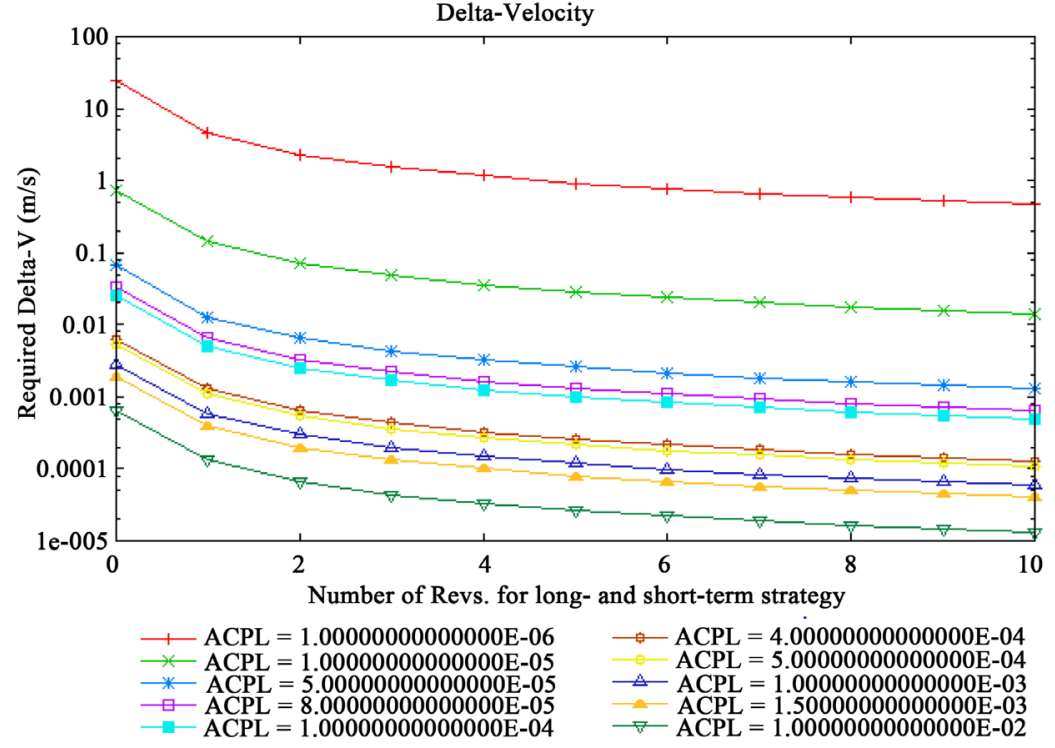

Figure 11. Required $\Delta V$ for OSIRIS $3 U$. 
It is observed that when sooner the manoeuvres are performed the more the cheaper they are. However, manoeuvres that performed much before the close approach have large uncertainties associated. The less the time to an event, the better the uncertainties are known, and therefore, the better the prediction.

\subsubsection{Propellant Mass Fraction for Avoidance Manoeuvres}

The propellant mass fraction burned during the expected avoidance manoeuvres, during the satellite lifetime, is linked to the required $\Delta V$ to perform those manoeuvres and the propulsion system characteristics.

The computation of the propellant mass fraction requires the specific impulse $\left(I_{s p}\right)$. The ratio between the propellant mass $\left(m_{p}\right)$ to be burned by a known $\Delta V$ and the initial satellite mass $\left(m_{o}\right)$ is given by:

$$
P M F_{a m}=\frac{m_{p}}{m_{o}}=\left[1-e^{\frac{-\Delta V}{I_{s p} g}}\right]
$$

Figure 12 shows the propellant mass fraction required to reach the ACPL values.

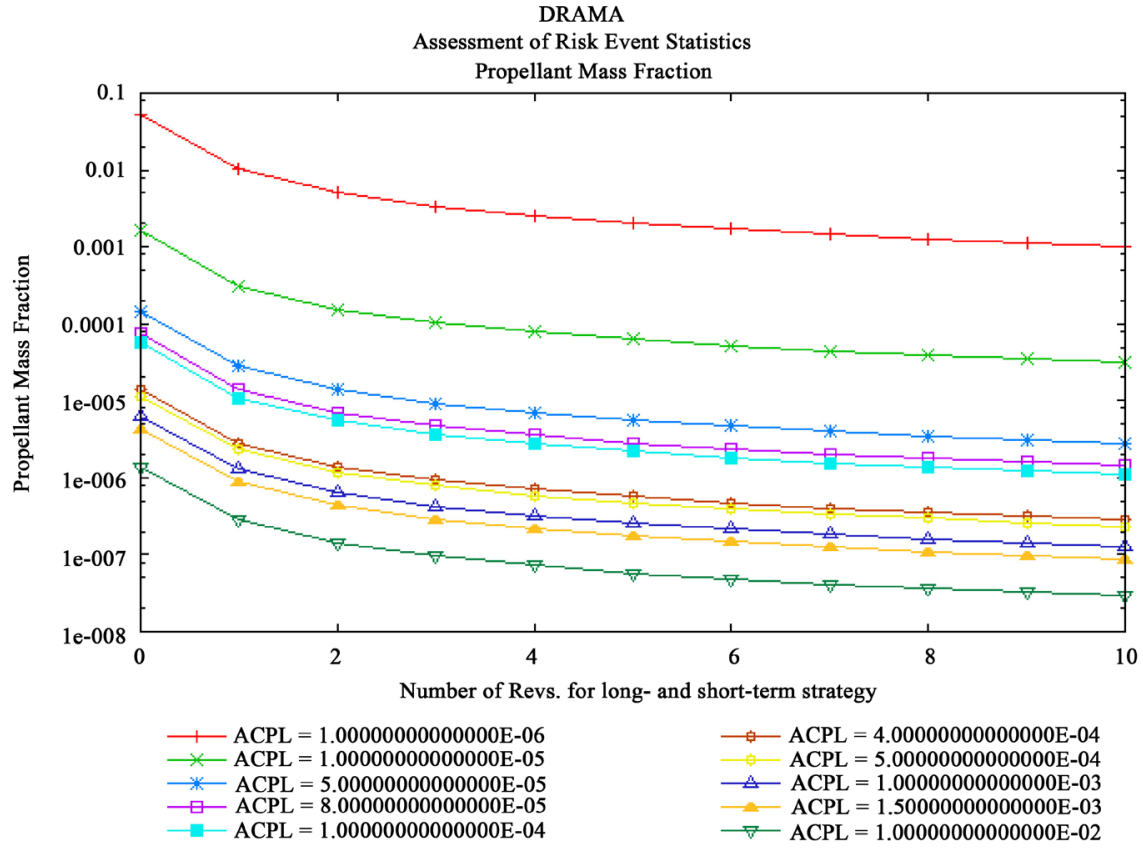

Figure 12. Required propellant mass for OSIRIS $3 \mathrm{U}$.

\subsection{MIDAS}

For the given simulation time $\Delta t$ and the impact flux $F$, the number of impacts $\left(n_{\text {imp }}\right)$ and the probability of collision $\left(P_{\text {coll }}\right)$ was computed.

Number of Impacts (NOI) $-n_{\text {imp }}=F \cdot A \cdot \Delta t$

Probability of collision (POC) $-P_{\text {coll }}=1-e^{-n_{i m p}}$

The orbit of OSIRIS $3 \mathrm{U}$ is the target orbit and is defined by the following parameters: 
Semi-major Axis $(a)=6646.4 \mathrm{~km}$.

Eccentricity $(e)=8.39 \mathrm{E}-3$.

Inclination $(i)=51.6305 \mathrm{deg}$.

Right ascension of the ascending node $(\Omega)=162.247 \mathrm{deg}$.

Argument of Perigee $(\omega)=350.489 \mathrm{deg}$.

And the objects considered are of range:

0.1000E+00 m-Lower Threshold

0.1000E+03 m-Upper Threshold

The flux was considered for a diameter range $1 \mathrm{~mm}<d<20 \mathrm{~cm}$ for a time span between May 1st, 2001 and May 1st, 2050. All sources of debris and the meteoroid were also considered.

Figure 13, npen $(m)$ is the average number of penetrations of the respective oriented plate by all particles with

$$
m p>m
$$

where,

$m p=$ particle mass.

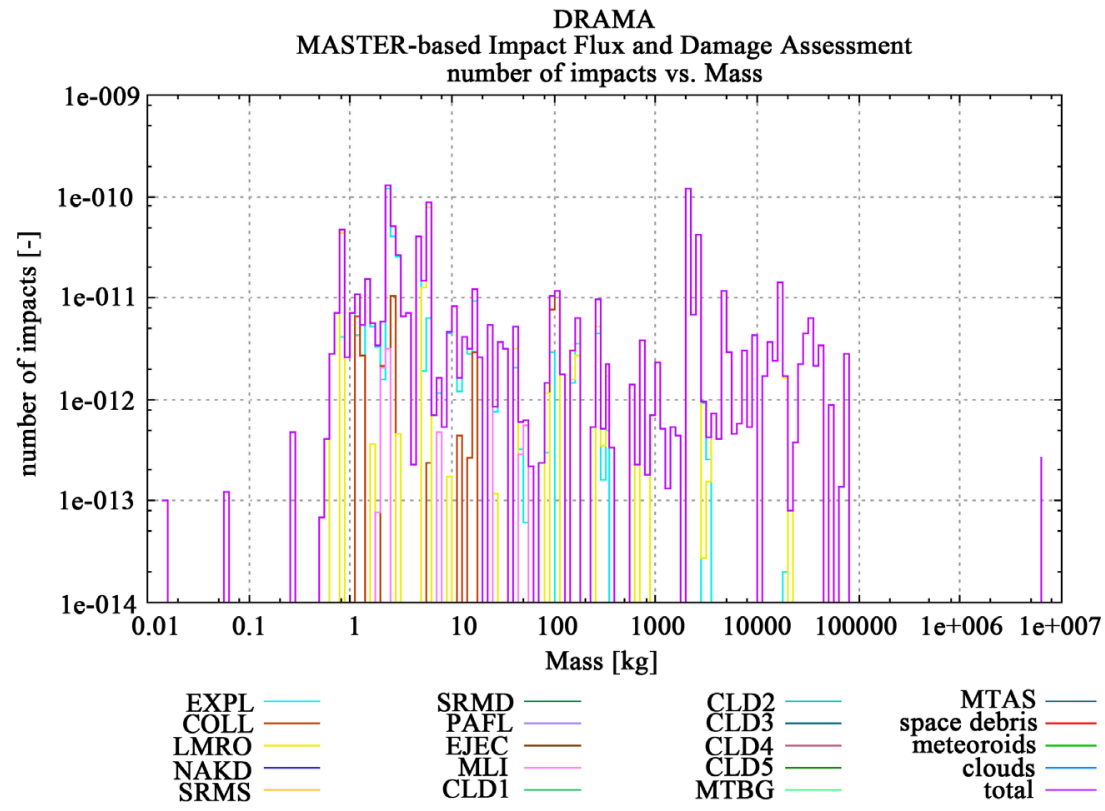

Figure 13. Mass vs number of impacts.

Figure 14 npen $(d)$ is the average number of penetrations of the respective oriented plate by all particles with

$$
d p>d
$$

where,

$d p=$ particle diameter.

Figure $15 \mathrm{PNP}(\mathrm{m})$ is the probability that no penetration of the oriented plate by a particle with $\mathrm{mp}>\mathrm{m}$ will occur. It is based on the average cumulated number of penetrations in this mass class. 
DRAMA

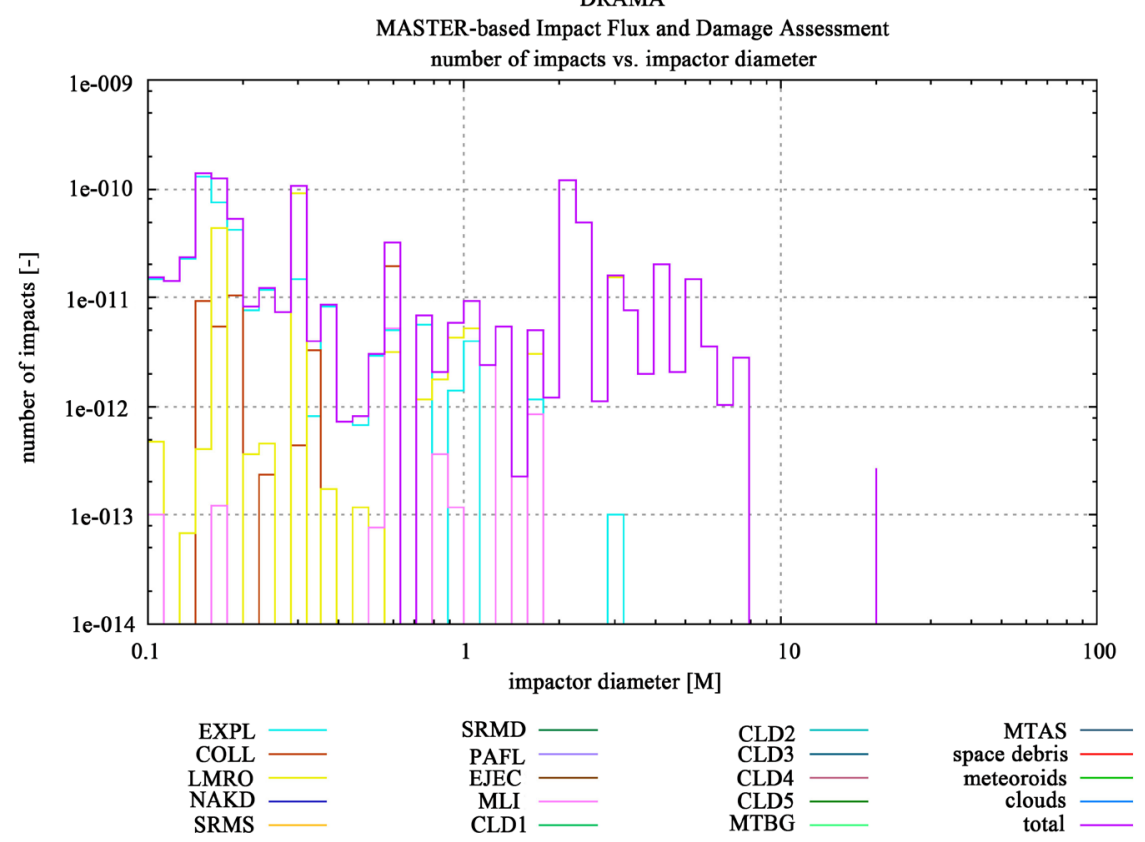

Figure 14. Impactor diameter vs number of impacts.

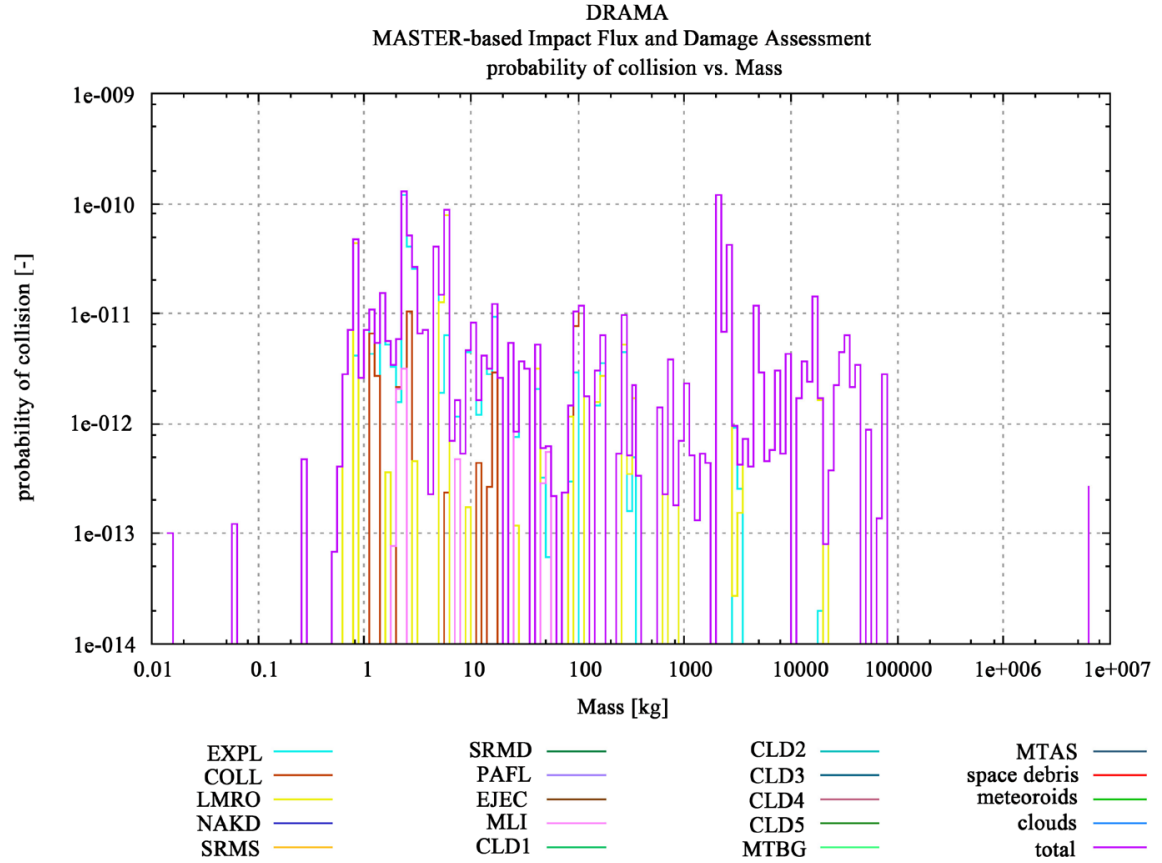

Figure 15. Mass vs probability of collision.

Figure 16, PNP $(d)$ is the probability that no penetration of the oriented plate by a particle with $d p>d$ will occur. It is based on the average cumulated number of penetrations in this diameter class.

On comparing the overall number of impacts on the reference sphere with those on the Sun oriented surface there are more impacts on the oriented surface than on the sphere. 


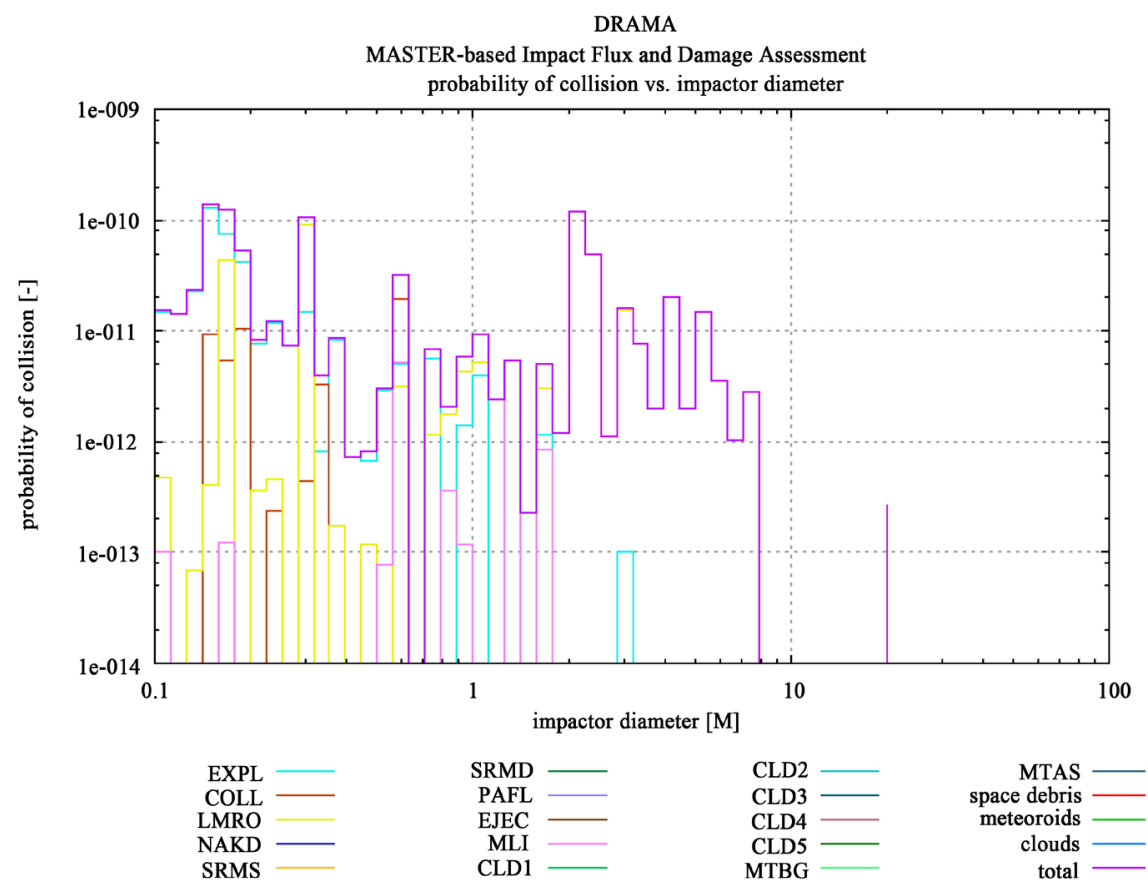

Figure 16. Impactor diameter vs probability of collision.

\subsection{OSCAR}

The residual lifetime of OSIRIS $3 \mathrm{U}$ was estimated by OSCAR for the minimum cross-section in flight direction. From Figure 17, it can be concluded that the end of orbital lifetime for this orbit was founded to be in May 2019, whereas the actual orbital lifetime ends in March 2019. The lifetime margin was considered as $1 \%$ and the disposal option was not considered. Figure 18, provides the evolution of singly averaged eccentricity of OSCAR and Figure 19, provides the evolution of singly averaged inclination of OSCAR.

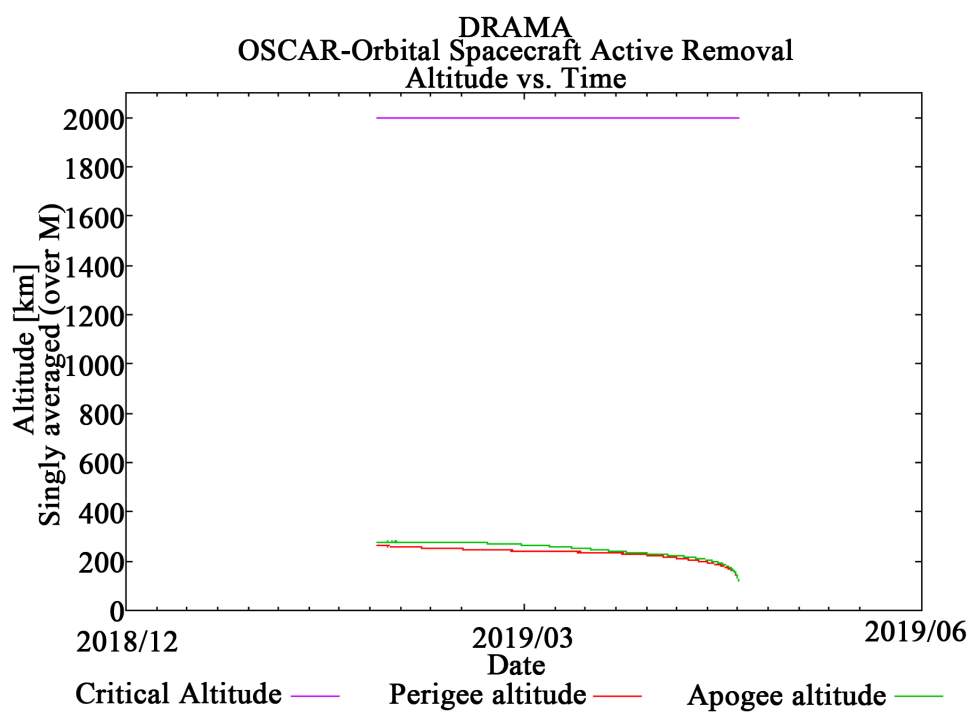

Figure 17. Date vs altitude. 


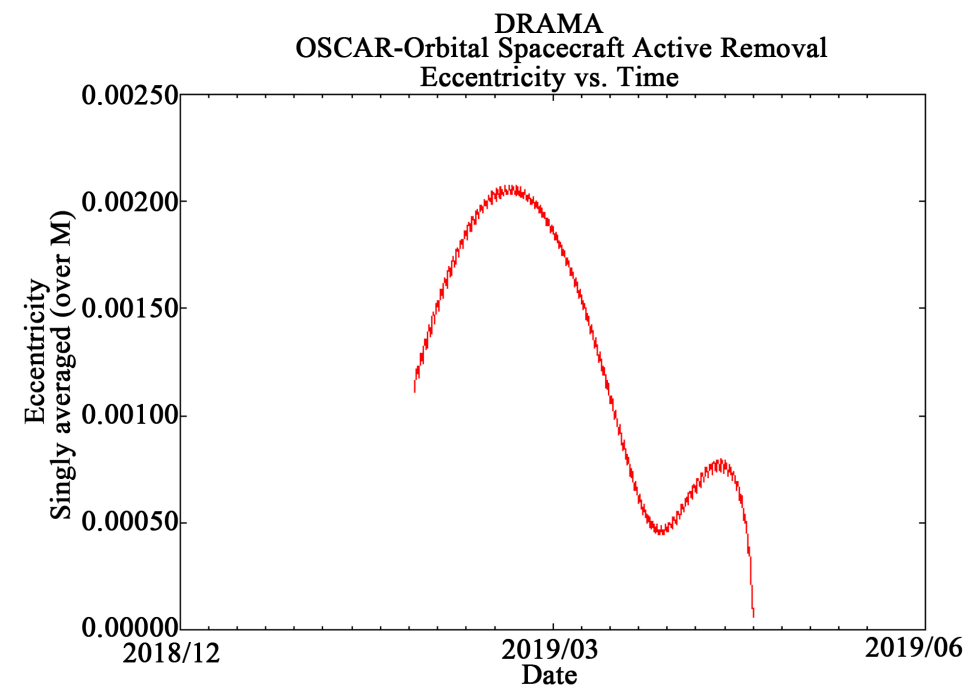

Figure 18. Date vs eccentricity.

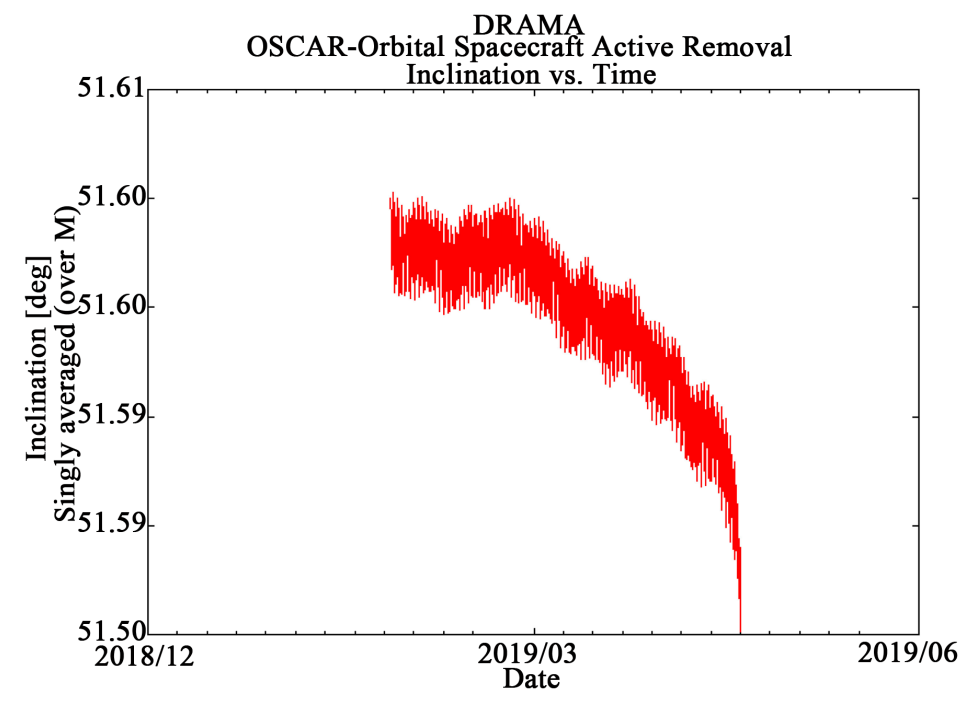

Figure 19. Date vs inclination.

\subsection{CROC}

The cross-sectional [9] computation is made using projections determined by using the given point of view direction and the Z-Buffer Algorithm for visible surface determination. The Z-Buffer Algorithm is an array of $n \times n$ of pixels. For each pixel there is a record of the depth of object within the pixel that lies closest to the observed. This method is used for hidden surface detection.

The cross section of a satellite with respect to the aspect angle $\theta=0, \varphi=0$ was found to be $19,900.0 \mathrm{~mm}^{2}$ and the perturbation of atmospheric drag acts on this surface.

For an angle of $\theta=45, \varphi=15$ the surface area is $45411 \mathrm{~mm}^{2}$.

\subsection{SARA}

Due to the large orbital velocity, the spacecraft experiences high mechanical 
loads and heating rates while entering the atmosphere and during the aero-braking process the potential and kinetic energy of the spacecraft is converted into thermal energy that is consumed by the spacecraft, resulting in thermal and mechanical loads destroying the spacecraft either completely or partially [6] [9] [10]. The spacecraft is destroyed either by melting or evaporation or chemical reaction. For thermal or mechanical destruction, the properties of the spacecraft materials are considered.

Object-oriented method was used to analyse and the breakup altitude was set for a range between $75 \mathrm{~km}$ and $85 \mathrm{~km}$. The solar panel breakup altitude was assumed to be at $95 \mathrm{~km}$.

Along with the re-entry trajectory dynamics, the aerodynamic, aero thermodynamic and thermal analyses were also performed for OSISRIS $3 \mathrm{U}$ re-entry. The initial conditions of the trajectory [11] and the spacecraft model was defined in terms of osculating orbital elements and by the spacecraft components, specified by their shape, size and material. The output of the analysis comprises the mass, cross-section, velocity, incident angle and impact location.

The trajectory path was found to be 51.5699 deg. (Lat), -86.5738 deg. (long.) with an altitude of $168.643 \mathrm{~km}$ at a velocity of $7.51313 \mathrm{~km} / \mathrm{s}$ with an approximate temperature of $300 \mathrm{~K}$.

It was found that no objects survived upon re-entry and so there was no ground impact.

\section{Conclusion}

The predicted re-entry time was found to be on 7th March 2019, 7:25 (UTC), whereas the actual re-entry time was on 7th March 2019, 7:03 (UTC). The trajectory path found was 51.5699 deg. (Lat), $-86.5738 \mathrm{deg}$. (Long.) with an altitude of $168.643 \mathrm{~km}$. But the actual trajectory was $51.76 \mathrm{deg}$. (Lat), $-89.01 \mathrm{deg}$. (Long.) with an altitude of $143.5 \mathrm{~km}$.

\section{Conflicts of Interest}

The authors declare no conflicts of interest regarding the publication of this paper.

\section{References}

[1] Sharma, R.K. (1990) On Mean Orbital Elements Computation for Near Earth Objects. Indian Journal of Pure and Applied Mathematics, 21, 468-474.

[2] Sharma, R.K. and Mani, L. (1985) Study of RS-1 Orbital Decay with KS Differential Equations. Indian Journal Pure and Applied Mathematics, 6, 833-842.

[3] Brauna, V., Gelhaus, J., Kebschull, C., Sanchez-Ortiz, N., Oliveira, J., Dominguez, R., Wiedemann, C., Krag, H. and Vorsmann, P. (2013) DRAMA 2.0-ESA's Space Debris Risk Assessment and Mitigation Analysis Tool Suite. 64th International Astronautical Congress, Beijing, September 2013.

[4] Hintz, G.R. (2015) Orbital Mechanics and Astrodynamics Techniques and Tools for Space Missions. Springer, Berlin, 24-26. 
[5] Mutyalarao, M. and Sharma, R.K. (2010) Optimal Re-Entry Time Estimation of an Upper Stage from Geostationary Transfer Orbit. Journal of Spacecraft and Rockets, 47, 686-690. https://doi.org/10.2514/1.44147

[6] Kummer, A.T. (2012) System Design and Instrumentation Development for the Osiris-3U CubeSat Mission. MS Thesis, The Pennsylvania State University, State College.

[7] Perini, L.L. (1974) Orbital Lifetime Estimates. The John Hopkins University, Baltimore, ANSP-M-8, Copy 55. https://doi.org/10.2172/4269348

[8] Gondelach, D.J., Armellink, R. and Lidtke, A.A. (2017) Ballistic Coefficient Estimation for Reentry Prediction of Rocket Bodies in Eccentric Orbits Based on TLE Data. Hindawi Mathematical Problems in Engineering, 2017, Article ID: 7309637. https://doi.org/10.1155/2017/7309637

[9] Garzon, M.M. (2012) Development and Analysis of the Thermal Design for the Osiris-3U CubeSat Mission. The Pennsylvania State University, State College.

[10] Song, S., Kim, H. and Chang, Y.-K. (2018) Design and Implementation of 3U CubeSat Platform Architecture. International Journal of Aerospace Engineering, 2018, Article ID: 2079219. https://doi.org/10.1155/2018/2079219

[11] De Lafontaine, J. and Garg, S.C. (1982) A Review of Satellite and Orbit Decay Prediction. Proceedings of the Indian Academy of Sciences, 5, 197-258. 